\title{
Avaliação das Heteroses Materna e Individual sobre o Ganho de Peso do Nascimento ao Desmame em Bovinos Angus x Nelore
}

\author{
Vanerlei Mozaquatro Roso1, Luiz Alberto Fries ${ }^{1,2}$
}

\begin{abstract}
RESUMO - Dados de 17.796 bovinos Angus x Nelore pertencentes a 50 rebanhos associados ao "Programa Natura", localizados nos Estados de Goiás, Minas Gerais, Mato Grosso, Mato Grosso do Sul, Paraná, Rio Grande do Sul, São Paulo e Tocantins foram analisados para estimar os efeitos das heteroses materna e individual sobre o ganho de peso do nascimento ao desmame. O modelo estatístico utilizado nas análises continha os efeitos fixos da média geral, grupo contemporâneo, heterozigoses materna e individual (covariáveis), efeitos aleatórios de touro (aditivo), vaca (efeito materno composto), segregação mendeliana e resíduo. Para estimar os efeitos, utilizou-se um procedimento iterativo, que alternava análises genéticas para grupos contemporâneos e efeitos aditivos+maternos sobre o ganho de peso pré-ajustado para heteroses e análises, com o objetivo de estimar os efeitos das covariáveis sobre o ganho de peso pré-ajustado para grupo contemporâneo e efeitos aditivos+maternos. O processo foi finalizado quando as estimativas se tornaram estáveis. Os resultados indicaram relação linear positiva entre as heterozigoses e as heteroses materna e individual. Foram obtidos valores de $15,46 \%$ (22,62 kg) para heterose materna e 9,10\% (13,31 kg) para heterose individual. Com base no grau de dificuldade para estimar os efeitos de heterose no presente trabalho, é necessário precaução ao sugerir a simples inclusão das heterozigoses no modelo estatístico e deixar que o ajuste se realize automaticamente.
\end{abstract}

Palavras-chave: bovinos de corte, cruzamentos, ganho de peso, hererozigose

\section{Evaluation of Maternal and Individual Heterosis on Preweaning Gain of Angus x Nellore Calves}

\begin{abstract}
Records from 17,796 Angus x Nellore cattle out of 50 Associated Herds from the "Program Nature", in the states of Goiás, Minas Gerais, Mato Grosso, Mato Grosso do Sul, Paraná, Rio Grande do Sul, São Paulo and Tocantins were analyzed in order to estimate the effects of maternal and individual heterosis on the weight gain from birth to weaning. The used statistical model included the fixed effects of a general mean and contemporary groups, maternal and individual heterozygosity (covariables), random effects of sire (additive), dam (composite maternal), mendelian segregation and residue. To estimate the effects, an iterative process was used, that alternated genetic analysis for the contemporary groups and additive + maternal effects on the readjusted weight gain for heterosis and statistical analysis to estimate the effects of the covariables on readjusting weight gain for the contemporary groups and additive + maternal effects. The process was ended when the estimates became stable. The results indicated a positive linear relationship between heterozygosity and maternal and individual heterosis. Estimated values of $15.46 \%$ (22.62 kg) for maternal heterosis and 9.10\% (13,31 kg) for individual heterosis were observed. Based on the degree of difficulties to estimate the heterosis effects in the present work, it is necessary caution to suggest just the inclusion of heterozygosities in the statistical model and let it be adjusted by itself.
\end{abstract}

Key Words: beef cattle, crosses, heterosis, heterozygosity, weight gain

\section{Introdução}

O aumento da competitividade é uma realidade na pecuária de corte brasileira. A busca por animais economicamente produtivos tem levado os criadores a investirem mais na aplicação de tecnologias relacionadas ao melhoramento genético. Nesse contexto, principalmente quando aliado à seleção, o cruzamento é um dos meios mais eficazes para incrementar a produtividade.

A superioridade dos animais cruzados em relação à média da contribuição parental, de raças puras, decorre da heterose ou do vigor híbrido e da possibilidade de complementaridade entre as raças. Como regra, características menos herdáveis, como aquelas relacionadas à adaptação e ao complexo reprodutivo, respondem melhor ao cruzamento, evidenciando a ação gênica não-aditiva.

O grau de heterose obtido nos cruzamentos depende do nível de heterozigose materna e individual, do distanciamento genético entre as raças envolvidas, das freqüências gênicas na população, da 


\section{ROSO e FRIES}

característica em questão e das interações com o ambiente (FRIES, 1996a). Os cruzamentos Bos indicus x Bos taurus resultam em maior heterose, comparativamente aos cruzamentos Bos taurus $\mathrm{x}$ Bos taurus. Para incrementar a eficiência da seleção em populações de animais cruzados, as características avaliadas também devem ser adequadamente ajustadas para os efeitos de heterose.

O objetivo do presente trabalho foi identificar o tipo de associação entre heterozigose e heterose, bem como estimar os efeitos das heteroses materna e individual sobre o ganho de peso do nascimento ao desmame, em animais Angus x Nelore, participantes de um programa de melhoramento genético.

\section{Material e Métodos}

Dados de ganho de peso do nascimento ao desmame (GND) de bovinos Angus x Nelore foram coletados em 50 rebanhos, participantes do Programa Natura, localizados nos Estados de Goiás, Minas Gerais, Mato Grosso, Mato Grosso do Sul, Paraná, Rio Grande do Sul, São Paulo e Tocantins. Na edição dos arquivos, partiu-se de uma base de dados com 42.444 animais, nascidos no período de 1985 a 1995 , sobre a qual foram aplicados os seguintes critérios de eliminação: animais com paternidade desconhecida (reprodutor múltiplo), grupos contemporâneos (GCs) com menos de 10 animais, GCs desconectados geneticamente à base de dados principal e classes de combinações de heterozigoses materna e individual com menos de 10 animais. O arquivo final, utilizado nas análises, continha 17.796 animais, filhos de 225 touros e 16.035 vacas, distribuídos em 513 GCs. Os grupos contemporâneos (GC) foram formados por animais de mesma fazenda, ano e estação de nascimento, sexo, grupo de manejo e data da pesagem ao desmame.

Como critério mínimo de estimabilidade, é necessário que cada GC contenha pelo menos duas classes de heterozigose das vacas e uma classe de heterozigose dos produtos ou vice-versa. Caso contrário, os dois efeitos ficam confundidos e GCs deste tipo não contêm nenhuma informação que auxilie a estimar as heteroses.

A composição racial dos animais de cada rebanho depende de uma combinação de clima, condições nutricionais e de manejo e objetivos comerciais de cada rebanho (fazenda). Nas regiões mais ao sul do Brasil, predominam animais com maior fração da raça Angus, ao passo que, nas demais regiões, os animais apresentam maior fração da Nelore. Na Tabela 1, é apresentada a distribuição dos animais, conforme a composição racial, expressa pela fração Nelore, dos touros (pais) e das vacas (mães).

A fórmula mais difundida na literatura internacional para calcular a retenção da heterozigose $(\mathrm{H})$ em gerações avançadas de compostos é dada por $\mathrm{H}=1-\sum_{i=1}^{n} P_{i}^{2}$, em que n é o número de raças utilizadas e $\mathrm{P}_{\mathrm{i}}$, a contribuição da i-ésima raça na composição genética dos produtos. Esta fórmula corresponde ao complemento da homozigose e pode ser utilizada com precisão quando todas as raças são de um mesmo grupo. Entretanto, considerando que diferentes heteroses existem mesmo entre raças taurinas, uma fórmula mais específica deve ser utilizada.

A Tabela 2 indica como as fórmulas para cálculo de heterozigoses foram desenvolvidas. O exemplo baseia-se em uma população composta, cuja composição genética é dada por $50 \%$ da raça $\mathrm{A}, 25 \%$ da raça $\mathrm{B}$ e $25 \%$ da raça $\mathrm{C}$. Para um gameta médio desta população e para determinado locus, existe uma probabilidade de $50 \%$ de que o gene presente seja

Tabela 1 - Distribuição dos animais conforme composição racial dos touros e vacas Table 1 - Distribuition of animals according to the genetic compositions of sires and dams

\begin{tabular}{lrrrrrrr}
\hline $\begin{array}{l}\text { Touros } \\
\text { Sires }\end{array}$ & \multicolumn{9}{c}{$\begin{array}{c}\text { Vacas } \\
\text { Dams }\end{array}$} \\
\cline { 2 - 8 } & Angus & $3 / 8$ & $1 / 2$ & $5 / 8$ & $3 / 4$ & $\begin{array}{l}\text { Nelore } \\
\text { Nellore }\end{array}$ \\
\hline Angus & 2065 & - & 66 & 247 & 42 & 4943 & 7363 \\
$3 / 8$ & 32 & 2312 & 250 & 84 & - & 4673 & 7351 \\
$1 / 2$ & - & - & - & - & - & 19 & 19 \\
$5 / 8$ & 381 & - & 68 & 151 & - & 58 & 658 \\
$3 / 4$ & 2367 & - & 38 & - & - & - & 2405 \\
Total & 4845 & 2312 & 422 & 482 & 42 & 9693 & 17796 \\
\hline
\end{tabular}


734 Rev. bras. zootec.

Tabela 2 - Probabilidade de ocorrência dos genes originários das raças $A, B$ e $C$ nos gametas de touros e vacas da população sintética $A B C$ (1/2A 1/4B 1/4C) e probabilidade de união destes genes

Table 2 - Occur probability of genes from breed $A, B$ and $C$ in the gametes of sires and dams in the sintetic population $A B C(1 / 2 A 1 / 4 B 1 / 4 C)$ and probability of union of thises genes

\begin{tabular}{lccc}
\hline $\begin{array}{l}\text { Touros } \\
\text { Sires }\end{array}$ & & $\begin{array}{c}\text { Vacas } \\
\text { Dams }\end{array}$ & \\
\hline $\mathrm{A}(0,50)$ & $\mathrm{AA}(0,25)$ & $\mathrm{AB}(0,125)$ & $\mathrm{AC}(0,125)$ \\
$\mathrm{A}(0,25)$ & $\mathrm{BA}(0,125)$ & $\mathrm{BB}(0,0625)$ & $\mathrm{BC}(0,0625)$ \\
$\mathrm{C}(0,25)$ & $\mathrm{CA}(0,125)$ & $\mathrm{CB}(0,0625)$ & $\mathrm{CC}(0,0625)$ \\
\hline
\end{tabular}

originário da raça $\mathrm{A}$, de $25 \%$ de que seja originário da raça $\mathrm{B}$ e de $25 \%$ de que seja originário da raça $\mathrm{C}$. $\mathrm{A}$ heterozigose resulta do pareamento de genes nãohomólogos de raças distintas. As probabilidades de formação deste tipo de pares de genes (A e B; A e C; e B e C) são apresentadas nas seis caselas fora da diagonal, ao passo que as probabilidades de genes homólogos encontradas no mesmo indivíduo são apresentadas na diagonal. No exemplo, a heterozigose é menor que 1,00 , porque genes de mesma origem podem se encontrar com probabilidades de $25 \%$ para os AA e de 6,25\% para os BB e igualmente para os CC (gerando uma homozigose de 37,5\%), conforme pode ser observado nas caselas da diagonal. Como a soma de todas as caselas é 1,00 , é mais fácil calcular a heterozigose por [1-soma das caselas na diagonal] do que pela soma das todas as caselas fora da diagonal. De qualquer forma, a heterozigose retida nesta população seria de 0,625 ou $62,5 \%$. A fórmula tradicional para o cálculo da heterozigose, portanto, é apenas o complemento da diagonal da Tabela 2.

Supondo que A seja uma raça zebuína e B e C sejam raças taurinas, a Tabela 2 mostra que a heterozigose gerada entre zebuínos e taurinos $\left(\mathrm{H}_{\mathrm{ZT}}\right)$ é de 0,50 (soma das caselas $\mathrm{AB}, \mathrm{AC}, \mathrm{BA}$ e $\mathrm{CA}$ ), enquanto a heterozigose entre taurinos $\left(\mathrm{H}_{\mathrm{TT}}\right)$ é de 0,125 (soma das caselas BC e CB).

Para qualquer situação, as heterozigoses podem ser decompostas nos componentes $\mathrm{H}_{\mathrm{ZZ}}$ (zebuína $\mathrm{x}$ zebuína), $\mathrm{H}_{\mathrm{TT}}$ (taurina $\mathrm{x}$ taurina) e $\mathrm{H}_{\mathrm{ZT}}$ (zebuína $\mathrm{x}$ taurina), de acordo com as fórmulas abaixo, reportadas por BERTOLI (1991), e com o desenvolvimento completo em SCHENKEL (1993), em que $\mathrm{P}_{\mathrm{j}}$ e $\mathrm{P}_{\mathrm{k}}$ representam as participações porcentuais das $\mathrm{Z}$ raças zebuínas e das $\mathrm{T}$ raças taurinas.

$$
\begin{gathered}
\mathrm{H}_{\mathrm{ZZ}}=\left(\sum_{j=1}^{Z} P_{j}\right)^{2}-\sum_{j=1}^{Z} P_{j}^{2} \\
\mathrm{H}_{\mathrm{TT}}=\left(\sum_{k=1}^{T} P_{k}\right)^{2}-\sum_{k=1}^{T} P_{k}^{2} \\
\mathrm{H}_{\mathrm{ZT}}=2 *\left[\sum_{j=1}^{Z} P_{j}-\left(\sum_{j=1}^{Z} P_{j}\right)^{2}\right]
\end{gathered}
$$

Para cada animal, calcularam-se a heterozigose materna (HM) e a heterozigose individual (HI), cuja distribuição é apresentada na Tabela 3.

O GND utilizado nas análises foi pré-ajustado por meio de fatores de correção multiplicativos para idade do bezerro (205 dias), idade da vaca (7 anos) e data do nascimento (3 de setembro), conforme CAMPOS (1988).

O modelo estatístico utilizado nas análises foi:

$\mathrm{GNDA}=\mathrm{M}+\mathrm{GC}+\mathrm{T}+\mathrm{V}+\mathrm{SM}+\beta_{1} \mathrm{HM}+$ $\beta_{2} \mathrm{HI}+\mathrm{e}$

em que

GNDA = ganho de peso do nascimento ao desmame ajustado;

$\mathrm{M}=$ média geral;

GC = grupo contemporâneo;

$\mathrm{T}=$ efeito genético aditivo do touro;

$\mathrm{V}=$ efeito composto (genético aditivo e materno e ambiental permanente) da vaca;

$\mathrm{SM}=$ efeito da segregação mendeliana do animal;

$\beta_{1}$ e $\beta_{2}=$ coeficientes de regressão linear;

$\mathrm{HM}=$ heterozigose materna;

$\mathrm{HI}=$ heterozigose individual; $\mathrm{e}$

e $\quad$ resíduo.

Os efeitos foram estimados num processo iterativo, alternando análises com fins de avaliação genética (solução iterativa para um modelo animal reduzido com GC), após pré-ajustamento do GNDA para heteroses, seguidas de análises estatísticas para as covariáveis, sendo o GNDA pré-ajustado para GC, $\mathrm{T}$ e V. O processo foi finalizado quando as estimativas se tornaram estáveis. Todos os passos do processo de estimação foram descritos por MONJELÓ (1995) e WOLF (1996). 


\section{Resultados e Discussão}

As heteroses materna e individual influenciaram significativamente o ganho de peso do nascimento ao desmame, sendo linear a relação com as heterozigoses $(\mathrm{P}<0,01)$. Os desvios da linearidade não foram significativos. Foram obtidos valores de 15,46\% (22,62 kg) para heterose materna e $9,10 \%(13,31 \mathrm{~kg})$ para heterose individual.

A relação entre heterozigose e heterose depende da importância dos diferentes tipos de ação gênica não-aditiva: dominância, sobredominância e epistasia. Segundo GREGORY e CUNDIFF (1980), a linearidade entre heterozigose e heterose, em características determinadas por vários loci, ocorre quando a dominância é a principal causa da heterose.

Os valores encontrados no presente trabalho estão relativamente próximos aos valores médios de $14 \%$ para heterose materna e $12 \%$ para heterose individual, obtidos por MONJELÓ (1995), em uma revisão de vários trabalhos de cruzamentos Bos indicus x Bos taurus. Em outro trabalho, após analisar os resultados de grande número de experimentos de cruzamentos entre Bos indicus e Bos taurus, KOGER (1976) relatou superioridade de 10,8 e 22,6\% no peso ao desmame dos produtos $\mathrm{F} 1$ e filhos de vacas $F 1$, respectivamente, em relação às raças puras. No Brasil, BARBOSA e ALENCAR (1995) apresentaram uma síntese dos resultados obtidos no período de 1934 a 1994 . Os autores relataram a superioridade média dos pesos do nascimento à maturidade e ganhos de peso dos cruzados em relação a animais puros: $10,8 \pm 2,1 \%$ para animais $\mathrm{F} 1 ; 14,2 \pm 2,4 \%$ para animais $\mathrm{F} 2 ; 19,5 \pm 4,1 \%$ para animais resultantes de retrocruzamento; e $33,5 \pm 9,0 \%$ para animais cruzas de três raças.

A heterose materna foi $69,89 \%$ superior à heterose individual, quando considerado o mesmo nível de heterozigose. GREGORY e CUNDIFF (1980) relataram que $60 \%$ ou mais da heterose em características que contribuem para o peso ao desmame são atribuídos à heterose materna. Por sua vez, CUNDIFF et al. (1974), ao analisarem alguns resultados de cruzamentos, observaram incremento de $23 \%$ na quantidade de quilogramas de bezerros desmamados por vaca exposta à reprodução, devido a efeitos heteróticos na fertilidade e produção de leite das vacas e, também, ao aumento da sobrevivência e da taxa de crescimento dos bezerros.

Os percentuais de heterose total, resultantes do somatório das heteroses materna e individual, para cada nível de heterozigose, são apresentados na Tabela 4. Os valores mais elevados são observados quando fêmeas cruzadas foram utilizadas na produção, sendo $20,01 \%$ o valor máximo, obtido nos produtos com $50 \%$ de heterozigose individual e $100 \%$ de heterozigose materna.

Estes resultados mostram que a utilização de vacas cruzadas potencializa mais eficientemente o aproveitamento da heterose total possível de ser obtida nos cruzamentos. Entretanto, se uma medida mais completa fosse considerada para comparar a eficiência produtiva, a diferença entre vacas puras e cruzadas poderia ser ainda maior, pois, como regra, os efeitos heteróticos são mais pronunciados nas características relacionadas ao complexo reprodutivo e sobrevivência dos produtos. KOCH et al. (1989) relataram que a heterose aumenta a produção por vaca em torno de $23 \%$ em cruzamentos Bos taurus x Bos taurus, em áreas temperadas, e pelo menos $50 \%$ em cruzamentos Bos indicus x Bos taurus, em áreas subtropicais.

O maior nível de heterose observado nos cruza-

Tabela 3 - Distribuição dos animais conforme heterozigose materna $(H M)$ e heterozigose individual (HI)

Tabele 3 - Distribuition of animals according to the maternal heterozigosity (HM) and individual heterozigosity (HI)

\begin{tabular}{|c|c|c|c|c|c|c|c|c|c|}
\hline \multirow[t]{2}{*}{$\mathrm{HM}$} & \multicolumn{8}{|c|}{ HI } & \multirow[t]{2}{*}{ Total } \\
\hline & 0,00 & 0,375 & 0,469 & 0,50 & 0,531 & 0,625 & 0,750 & 1,00 & \\
\hline 0,00 & 2065 & 90 & - & 19 & - & 5054 & 2367 & 4943 & 14538 \\
\hline 0,375 & - & - & - & - & - & - & 42 & - & 42 \\
\hline 0,4688 & - & - & 2409 & - & 84 & 247 & - & - & 2740 \\
\hline 0,50 & - & - & - & 406 & - & - & - & - & 406 \\
\hline 0,75 & - & - & 54 & - & - & - & - & - & 54 \\
\hline 1,00 & - & - & - & 16 & - & - & - & - & 16 \\
\hline Total & 2065 & 90 & 2463 & 441 & 84 & 5301 & 2409 & 4943 & 17796 \\
\hline
\end{tabular}


Tabela 4 - Heterose total (\%) conforme heterozigose materna (HM) e heterozigose individual (HI)

Table 4 - Total heterosis (\%) according to maternal heterozigosity and individual heterozigosity (HI)

\begin{tabular}{lrrrrrrrrr}
\hline HM $(\%)$ & \multicolumn{9}{c}{$\mathrm{HI}(\%)$} \\
\cline { 2 - 8 } & 0,00 & 0,375 & 0,469 & 0,50 & 0,531 & 0,625 & 0,75 & 1,00 & Mean \\
\hline 0,00 & 0,00 & 3,41 & - & 4,55 & - & 5,69 & 6,83 & 9,10 & 4,93 \\
0,375 & - & - & - & - & - & - & 12,62 & - & 12,62 \\
0,4688 & - & - & 11,52 & - & 12,08 & 12,94 & - & - & 12,18 \\
0,50 & - & - & - & 12,28 & - & - & - & - & 12,28 \\
0,75 & - & - & 15,86 & - & - & - & - & - & 15,86 \\
1,00 & - & - & - & 20,01 & - & - & - & - & 20,01 \\
Média & 0,00 & 3,41 & 13,69 & 12,28 & 12,08 & 9,31 & 9,72 & 9,10 & 9,10 \\
Mean & & & & & & & & & \\
\hline
\end{tabular}

mentos de raças zebuínas com taurinas, em comparação aos cruzamentos entre raças taurinas, é largamente documentado na literatura (KOGER, 1980; MADRUGA et al., 1984; SALOMONI et al., 1987; GOMES et al., 1989; OLSON et al., 1991; e OLSON et al., 1993). Portanto, em determinados ambientes, a exploração racional dos cruzamentos Bos indicus x Bos taurus é uma oportunidade ímpar para a pecuária de corte promover um salto significativo em produtividade. Evidentemente, para obter os benefícios máximos de um programa de cruzamento, é necessário escolher raças que se complementem de forma efetiva nas características economicamente importantes; além disso, o produto resultante deve manter alto nível de produção econômica no sistema empregado.

Para manter ganhos contínuos em produtividade, os sistemas de cruzamentos devem estar aliados a eficientes programas de seleção. Trabalhos intensivos de formação de raças ou linhagens compostas mostraram tendência de perda das vantagens iniciais do cruzamento (da F1), à medida que as gerações avançam. Essa perda é atribuída à recombinação e à consangüinidade. Segundo DIKERSON (1973), a perda por recombinação é consequiência da destruição gradativa dos efeitos epistáticos. Os arranjos gênicos existentes nos cromossomos dos pais, intactos na geração $\mathrm{F}$, se desintegram progressivamente nas gerações sucessivas e, se a epistasia for importante, pode causar queda no desempenho. Ainda que uma fração apreciável dos efeitos que agem sobre características de produção seja do tipo aditivo, medida pela herdabilidade, perdas por recombinação acumulamse durante as primeiras gerações de uma raça composta, sendo necessária forte pressão de seleção para neutralizálas e aumentar a freqüência das combinações gênicas favoráveis. Ao mesmo tempo, é necessária uma população de grande tamanho para evitar o aparecimento e a acumulação dos efeitos depressivos da consangüindade com o passar das gerações.

Com base no grau de dificuldade encontrado para estimar os efeitos de heterose no presente trabalho, assim como nas experiências de MONJELÓ (1995) e WOLF (1996), é preciso precaução, ao sugerir a simples inclusão das heterozigoses no modelo estatístico e deixar que o ajuste se realize automaticamente. O conjunto de dados utilizados no presente trabalho provém de rebanhos com bom volume, planejamento, estrutura (mais de $70 \%$ dos dados são de inseminação artificial) e qualidade dos dados. Estimar heterose em dados não-experimentais não é um procedimento simples e não deve ser automatizado até que estudos sejam realizados em anos consecutivos e sinais de consistência nos resultados sejam observados.

\section{Conclusões}

As heteroses materna e individual influenciam significativamente o ganho de peso ajustado do nascimento ao desmame, sendo linear a relação com as heterozigoses.

As heteroses materna e individual sobre o ganho de peso ajustado donascimento ao desmame são de $15,46 \%$ $(22,62 \mathrm{~kg})$ e $9,10 \%(13,31 \mathrm{~kg})$, respectivamente.

A heterose materna é $69,89 \%$ superior à heterose individual, considerando um mesmo nível de heterozigose.

\section{Referências Bibliográficas}

BARBOSA, P.F. ALENCAR, M.M. Sistemas de cruzamentos em bovinos de corte: Estado da arte e necessidade de pesquisa. In: REUNIÃO ANUAL DA SOCIEDADE BRASILEIRA DEZOOTECNIA, 32, 1995. Brasília. Anais ... Brasília: SBZ, 1995. p.681-683. 
BERTOLI, C.D. Sistema Cruza - Controle de produção e avaliação dos valores genéticos dentro de uma população bovina sintética. Porto Alegre, RS: UFRGS, 1991.233p. Dissertação (Mestrado em Zootecnia) - Universidade Federal do Rio Grande do Sul, 1991.

CAMPOS, L.T., SILVA, P.R., FRIES, L.A. Fatores de correção para efeitos ambientais que afetam o ganho de peso do nascimento à desmama em bovinos da raça Nelore. In: CONGRESSO BRASILEIRO DE ZEBU, 1, 1988, Uberaba.Anais... Uberaba: Empresa de Pesquisa Agropecuária de Minas Gerais, 1988. p.108-123.

CUNDIFF, L.V., GREGORY, K.E., SCHWULST, F.J., KOCH, R.M. 1974. Effects of heterosis on maternal performance and milk production in Hereford, Angus and Shorthorn cattle. J.Anim.Sci., 38(4):728-745.

DIKERSON, G.E. Inbreeding and heterosis in animals. In: ANIMAL BREEDING AND GENETICS SYMPOSIUM IN HONOR OF DR. J. L.LUSH. 1973. Champaign, IL. Proceedings... Champaign, 1973. p.54-77.

FRIES, L.A. Calculando e decompondo heterozigoses. In: SIMPÓSIO NACIONAL DE MELHORAMENTO ANIMAL. 1, 1996, Ribeirão Preto. Anais... Ribeirão Preto: Sociedade Brasileira de Melhoramento Animal, 1996a. p.246248.

FRIES, L.A. Maximizar heterozigose ou heterose? In: SIMPÓSIO NACIONAL DE MELHORAMENTO ANIMAL, 1, 1996, Ribeirão Preto. Anais... Ribeirão Preto: Sociedade Brasileira de Melhoramento Animal, 1996b. p.252-254.

GOMES, A., HONER, M.R., SCHENK, M.A.M., CURVO, J.B.E. 1989. Populations of the cattle tick (B. micropolus) on purebred Nelore, Ibagé and Nelore x European crossbreds in the Brazilian savanna. Trop. Anim. Hlth. Prod., 21(1):20-24.

GREGORY, K.E., CUNDIFF, L.V. 1980. Crossbreeding in beef cattle: evaluation of systems, J. Anim. Sci., 51(5):1224-1242.

KOCH, R.M., CUNDIFF, L.V., GREGORY, K.E. Beef cattle breed resource utilization. In: UTILIZATION OF ANIMAL GENETIC RESOURCES IN LATIN AMERICA, 1989, Ribeirão Preto. Proceedings...Ribeirão Preto, 1989, p.55-77.

KOGER, M. 1976. Resumen y conclusiones. In: KOGER, M., CUNHA, T.J., WARNICK, A.C. (Eds.). Cruzamientos en ganado vacuno de carne. Montevideo, Hemisferio Sur. p.535-552.

KOGER, M. 1980. Effective crossbreeding systems utilizing zebu cattle. J.Anim.Sci. 50(6):1215-1220.

MADRUGA, C.R., AYCARDI, R., KESSLER, R.H. et al. 1984. Níveis de anticorpos anti-Babesia bigemina e Babesia bovis, em bezerros da raça Nelore, Ibagé e cruzamentos de Nelore. Pesq. Agropec. Bras., 19(9):1163-1168.

MONJELÓ, L.A.S. Avaliação da heterose do ganho de peso até o desmame e no pós-desmame em populações de gado de corte resultantes do cruzamento entre Bos taurus indicus e Bos taurus taurus. Porto Alegre, RS: UFRGS, 1995. 227p. Tese (Doutorado em Zootecnia) - Universidade Federal do Rio Grande do Sul, 1995.

OLSON, T.A., EUCLIDES FILHO, K., CUNDIFF, L.V., KOGER, M., BUTTS JR, W.T., GREGORY, K.E. 1991. Effects of breed group by location interaction on crossbred cattle in Nebraska and Florida. J. Anim. Sci., 69:104-114.

OLSON, T.A., PEACOCK, F.M., KOGER, M. 1993. Reproductive and maternal performance of rotational, threebreed and inter se crossbred cows in Florida. J. Anim. Sci., 71:2322-2329.

SALOMONI, E., CHAGAS, E.C., DEL DUCA, L.O.A. 1987. Formação e seleção para uma nova raça. Bagé: EMBRAPA - CNPO, 1(2):245-260.

SCHENKEL, F.S. 1993. Cálculo das heterozigoses. GenSys Consultores Associados S/C Ltda, Porto Alegre, RS. 3p.

WOLF, P.G.L. Efeitos da heterozigose individual e materna sobre o ganho de peso do nascimento ao desmame de terneiros Pampiano-Braford. Porto Alegre, RS: UFRGS, 1996. 118p. Dissertação (Mestrado em Ciências Veterinárias) - Universidade Federal do Rio Grande do Sul, 1996.

Recebido em: 12/05/98

Aceito em: 05/10/99 